\title{
Derechos humanos desde la diversidad lingüística e identitaria de las personas sordas
}

\author{
Human rights from the perspective of deaf people's \\ linguistic and identity diversity
}

\author{
Amaia Jauregi \\ BAM Escuela Universitaria de Magisterio \\ ajauregi@bam.edu.es \\ Elena Bernarás \\ Universidad del País Vasco/Euskal Herriko Unibertsitatea \\ elena.bernaras@ehu.eus \\ Joana Jaureguizar \\ Universidad del País Vasco/Euskal Herriko Unibertsitatea \\ joana.jauregizar@ehu.eus
}

Cómo citar/Citation: Jauregi, Amaia, Elena Bernarás y Joana Jaureguizar. 2020. «Derechos humanos desde la diversidad lingüística e identitaria de las personas sordas». Deusto Journal of Human Rights, n. 5: 187-213. doi: http://dx.doi.org/10.18543/djhr.1748

Sumario: 1. Evolución del concepto de discapacidad en el contexto español. 2.Contextos de exclusión de las personas sordas en el Estado español. 2.1. El desconocimiento de su realidad y de su identidad. 2.2. Dificultades de accesibilidad. A modo de conclusión. Referencias bibliográficas.

Resumen: Este artículo pretende contribuir a la toma de conciencia sobre la diversidad lingüístico-cultural de las personas sordas y sus necesidades, así como sobre la situación de exclusión que vivencian en todas las áreas de la vida debido, por un lado, al desconocimiento social de sus características y necesidades y, por otro, a las barreras de comunicación y de acceso a la información. Las personas sordas se encuentran en una situación de discapacidad socialmente desapercibida. Para conocer su realidad, se muestran algunas pinceladas sobre sus características audiológicas y lingüístico-culturales, y se exponen algunos hechos y situaciones que explicitan las barreras sociales y actitudinales a las que se enfrentan en diversos ámbitos de su vida cotidiana. De esta manera se pretende evidenciar 
la vulneración de los derechos que recoge la legislación vigente y la situación de discriminación en la que viven.

Palabras clave: Discapacidad, derechos humanos, sordera, cultura sorda, identidad de las personas sordas

Summary: This article seeks to raise social awareness of the linguistic and cultural diversity of deaf people and their needs, as well as of the situation of social exclusion they experience in all areas of life. This is due, on the one hand, to widespread ignorance of their characteristics and their needs and, on the other hand, to the barriers they encounter in their access to information and communication. Society is unaware of the implications of deaf people's disability. This article explores some of their audiological, linguistic and cultural characteristics, as well as events and situations that show the social and attitudinal barriers they come across in their everyday life. We intent to show that their situation entails a violation of fundamental rights present in current legislation and discrimination against the group.

Keywords: disability, human rights, deafness, deaf culture, deaf people's identity 


\section{Evolución del concepto de discapacidad en el contexto español}

El fenómeno de la discapacidad es complejo, ya que en él interviene la combinación de multitud de factores que abarcan desde las propias diferencias y experiencias individuales hasta las percepciones y actitudes hacia la discapacidad que están sujetas a interpretaciones en cada contexto físico, social y cultural.

Durante muchos años, la discapacidad ha sido entendida como un problema individual que afecta a algunas personas como consecuencia de una deficiencia, pero, además, también puede ser entendida como construcción social; es decir, se construye en el día a día en la interacción entre las personas y los grupos sociales, en sus actitudes, decisiones y relaciones $y$, en la forma en que continuamente se va estructurando el entorno físico, social, cultural e ideológico en el que conviven (Barton 1998, Joly 2002).

A lo largo de la historia, la consideración de la dignidad humana, el derecho a la educación y la formación, el acceso al empleo y la participación social de las personas en situación de discapacidad han seguido la evolución de las mentalidades sociales en diferentes épocas y contextos.

Aunque la versión más extendida de la historia de la discapacidad ha sido explicada por numerosos autores planteando la dialéctica entre el modelo médico y el modelo social, más allá de ello, el concepto de discapacidad se ha ido definiendo desde nuevas perspectivas (OMS 2001, Palacios 2008, Toboso y Guzmán 2010).

El planteamiento explicativo de la discapacidad desde el modelo social ha ido evolucionando, ganando espacio la concepción de la persona con discapacidad desde un enfoque biopsicosocial (OMS 2011, Padilla 2010), y como una cuestión de derechos humanos (Asís 2014, CERMI 2019, Lidón 2016). Y desde el reconocimiento de que todos los seres humanos tienen el mismo valor y los mismos derechos, se reivindica la consideración del valor inherente de la persona por su diversidad, y la necesidad de que las políticas públicas se centren en hacer desaparecer la discapacidad, aunque existan las deficiencias, permitiendo de este modo a las personas con discapacidad su condición de ciudadanía (Palacios y Romañach 2006, CERMI 2009, Romañach 2009, Díez Velázquez 2017).

La OMS (2001) define la discapacidad desde un enfoque biopsicosocial, como un fenómeno complejo, dinámico y multidimensional, en el que intervienen factores personales (limitaciones en la actividad y restricciones en la participación) y factores contextuales (barreras y obstáculos). Los factores personales constituyen el trasfondo 
particular de cada persona y su estilo de vida, y se refieren a factores como la edad, el sexo, el nivel social, experiencias vitales, estilos de vida, hábitos, estilos de afrontamiento, la educación, la profesión, patrones globales de comportamiento, etc. En cuanto a los factores ambientales, se refieren a todos los aspectos del contexto de la vida de una persona, que afectan a su funcionamiento: el ambiente físico, social, actitudinal, relacional, incluyendo los valores, servicios y sistemas sociales y políticos, normas y leyes de la sociedad en la que vive. Estos factores ambientales interactúan con los componentes de funcionamiento (estructuras y funciones corporales, actividades y participación) y pueden actuar como barreras o facilitadores (OMS 2011). Entre los factores ambientales en el entorno de una persona que condicionan el funcionamiento y crean discapacidad se pueden incluir aspectos como, por ejemplo, un ambiente físico inaccesible, la falta de tecnología asistencial apropiada, las actitudes negativas respecto a la discapacidad, así como la falta de servicios, sistemas y políticas que favorezcan la participación. Entre los factores facilitadores, cuya presencia en el entorno de una persona mejora el funcionamiento y reduce la discapacidad, se consideran, por ejemplo, la existencia de un ambiente accesible, la disponibilidad de la tecnología necesaria, las actitudes positivas de la población hacia la discapacidad, las relaciones y el apoyo en la familia, los amigos, un adecuado entorno laboral, o la disponibilidad de servicios, sistemas y políticas que propicien la participación de las personas con una condición de salud en todas las áreas de la vida. Igualmente, la ausencia de un factor puede ser un facilitador, como por ejemplo, la ausencia de estigmas o actitudes negativas entre la población.

Con este planteamiento, el enfoque biopsicosocial tiene en cuenta la perspectiva biológica e individual defendida desde el modelo médico y, a su vez, respalda los planteamientos del modelo social teniendo en cuenta el conjunto de condiciones creadas en gran parte por el entorno. Se parte, por tanto, de la base conceptual de que los problemas a los que se enfrenta una persona con una discapacidad no son esencialmente médicos (Zola 2005). El entorno social, actitudinal, arquitectónico, médico, económico y político no se adecúa a las necesidades de todas las personas, por lo que las barreras sociales, obstáculos estructurales, organizativos y relacionales, son los causantes de la situación de desventaja y desigualdad en que se encuentran (Susinos 2006). Es decir, no se niega la existencia de unas condiciones biológicas, pero lo que define a la persona con discapacidad son las características del entorno (Asís 2013, Díaz Velázquez 2010). 
Desde este planteamiento se defiende que, para que todas las personas, incluidas las personas en situación de discapacidad, puedan participar en todas las áreas de la vida, es preciso modificar los factores ambientales y, para ello, es necesaria la implicación y responsabilidad colectiva de toda la sociedad. En este sentido, la discapacidad se plantea como un tema de índole política, en el que estos cambios sociales constituyen una cuestión de derechos humanos (OMS 2001, CERMI 2019). En esta línea, paralelamente a la transformación de los modelos explicativos de la discapacidad, y con el fin de lograr la inclusión de todas las personas garantizando el principio de igualdad de oportunidades, se han ido buscando respuestas basadas en el respeto a los valores que fundamentan los derechos humanos (Asís 2014).

Esta misma evolución en la consideración de la discapacidad se ha visto reflejada en la legislación española. La Ley 13/1982, de 7 de abril, de Integración Social de los Minusválidos (LISMI) fue la primera ley que reguló la atención y los apoyos a las personas con discapacidad y sus familias conforme a la Constitución. Esta ley reconoció a las personas con discapacidad como titulares de derechos, pero lo hizo desde la concepción de la discapacidad desde un enfoque médico, tal y como se recoge en su artículo 7: "se entenderá por minusválidos toda persona cuyas posibilidades de integración educativa, laboral o social se hallen disminuidos como consecuencia de una deficiencia, previsiblemente permanente, de carácter congénito o no, en sus capacidades físicas, psíquicas o sensoriales». Si bien supuso un primer avance en cuanto a que reconoce que las posibilidades de integración educativa, laboral y social están disminuidas, se entendía que ello era debido a su deficiencia y, por tanto, se estableció una serie de prestaciones económicas, servicios, medidas de integración laboral, accesibilidad y principios que más adelante fueron introducidos al ámbito de la sanidad, la educación y el empleo.

En el ámbito nacional español, el modelo social fue reconocido dos décadas más tarde, en la Ley 51/2003, de 2 de diciembre, de Igualdad de Oportunidades, no discriminación y accesibilidad universal de las personas con discapacidad (LIONDAU). Esta ley se concibió fundamentándose en los principios de vida independiente, normalización, accesibilidad universal, diseño para todos, diálogo civil y transversalidad de las políticas en materia de discapacidad. En la exposición de motivos se explicaba que las desventajas que vivían las personas con discapacidad eran debidas tanto a sus dificultades personales como a los obstáculos y condiciones sociales limitadoras de su plena participación, aludiendo a que estas condiciones ambientales habían sido creadas por la propia sociedad; una sociedad diseñada y 
proyectada al servicio de un modelo de persona media. Desde esta perspectiva, algunas de las disposiciones finales que se propusieron fueron las siguientes: exigencias de accesibilidad, condiciones favorables para el acceso, participación y utilización de recursos, apoyos complementarios como, por ejemplo, ayudas económicas, ayudas técnicas, asistencia personal, servicios especializados, y ayudas y servicios auxiliares para la comunicación como apoyos a la comunicación oral y lengua de signos $u$ otros dispositivos para posibilitar la comunicación.

Sin embargo, el modelo social de derechos humanos se consolidó tras la aprobación de la Convención sobre los Derechos de las Personas con Discapacidad (CDPD), el primer tratado internacional del siglo XXI, aprobado por la Asamblea General de la ONU en el año 2006 y ratificado por España y en vigor desde mayo del año 2008. Esta Convención internacional tiene como finalidad garantizar el reconocimiento y ejercicio de los derechos humanos de las personas con discapacidad en todos los ámbitos de la vida (cultura, educación, salud, trabajo, ocio, participación social y económica...), y para ello la Convención definió la condición de persona con discapacidad aunando las dos dimensiones: por un lado, la dimensión biológica (el déficit en el funcionamiento físico, mental, intelectual o sensorial) y, por otro lado, la dimensión ambiental que impide la participación en igualdad de condiciones, es decir, las barreras legales, interpersonales, físicas, de comunicación, de accesibilidad, etc. que estas personas encuentran en el reconocimiento y ejercicio de sus derechos. Por tanto, inspirada en la filosofía del modelo social, la Convención instauró el enfoque de los derechos humanos y discapacidad, y reconoció sus derechos desde la igualdad, la no discriminación y la autonomía, partiendo de esta doble dimensión (Palacios 2008).

La CDPD se basa en los principios y valores que sustentan a los derechos humanos, como son: la dignidad de todos y cada uno de los seres humanos, el concepto de autonomía o de libre determinación, la igualdad inherente de todas la personas independientemente de las diferencias, la no discriminación, la accesibilidad, la igualdad de oportunidades, la plena participación y la ética de la solidaridad que la sociedad exige para sustentar la libertad de la persona con los apoyos sociales correspondientes. En consecuencia, se hace hincapié en la necesidad de desarrollar medidas para impulsar la participación y el bienestar de todas las personas con discapacidad (OMS 2011).

Desde su entrada en vigor, en España se han producido importantes avances en la política legislativa con la finalidad de adecuar el sistema jurídico español a la Convención, siguiendo las recomendaciones 
recogidas en el artículo 4.11. En esta línea, en el año 2011, se aprobó la Ley 26/2011, de 1 de agosto, de adaptación normativa a la Convención Internacional sobre los Derechos de las Personas con Discapacidad², que supuso un impulso importante en el proceso de adaptación del ordenamiento jurídico a los principios, valores y mandatos del Tratado. Y posteriormente, en aplicación de la disposición final segunda de esta Ley, se dictó el Real Decreto Legislativo 1/2013, de 29 de noviembre, por el que se aprueba el Texto Refundido de la Ley General de derechos de las personas con discapacidad y de su inclusión sociaß ${ }^{3}$. Un Real Decreto en el que se reconoce que la sociedad ha mantenido a las personas con discapacidad en condiciones de exclusión, restringiendo sus derechos básicos y libertades, debido a los obstáculos que se generan por la forma en que funciona y está estructurada la sociedad, y establece la necesidad de garantizar el derecho a la igualdad de oportunidades y de trato, así como el ejercicio real y efectivo de derechos por parte de las personas con discapacidad en igualdad de condiciones respecto del resto de ciudadanos y ciudadanas, a través de la promoción de la autonomía personal, de la accesibilidad universal, del acceso al empleo, de la inclusión en la comunidad y la vida independiente y de la erradicación de toda forma de discriminación, por un lado. Y, por otro lado, recoge el régimen de infracciones y sanciones que garantizan las condiciones básicas en materia de igualdad de oportunidades, no discriminación y accesibilidad universal de las personas con discapacidad.

Sin embargo, la Delegación del Comité Español de Representantes de Personas con Discapacidad (CERMI) para la Convención de la ONU y los derechos humanos ${ }^{4}$, en sus informes anuales, sigue destacando que los derechos humanos continúan siendo una realidad lejana para las personas con discapacidad (CERMI 2009, 2012, 2017, 2018), por

1 Artículo 4.1. "Los Estados Partes se comprometen a adoptar las medidas pertinentes, legislativas, administrativas y de otra índole, para modificar, derogar leyes, reglamentos, costumbres y prácticas discriminatorias, con la finalidad de que se cumplan los derechos reconocidos en la Convención».

2 Ver BOE núm. 184, de 2 de agosto de 2011.

3 Ver BOE núm. 289, de 3 de diciembre de 2013.

4 El 17 de septiembre de 2009, el Estado español designó, en cumplimiento de lo previsto en el apartado 33.2 de la CDPD, al CERMI como mecanismo independiente de la sociedad civil para el seguimiento de su aplicación en España. Designación que alcanzó rango normativo al ser establecida en virtud de lo contenido en la Disposición adicional primera del Real Decreto 1276/2011, de 16 de septiembre, de adaptación normativa a la Convención Internacional sobre los Derechos de las Personas con Discapacidad. CERMI elabora anualmente informes para la Convención de la ONU, disponibles en su página web: www.cermi.es/es/colecciones/onu 
lo que reivindica hacer efectivos los compromisos de los tratados y los dictámenes de derechos humanos ratificados por España, único país de Europa en el que no existe un Plan de Derechos Humanos (CERMI 2019, 22).

La sociedad española sigue estando diseñada de forma preferente para el disfrute de una parte de la población que, mayoritariamente, no tiene dificultades que le impidan acceder a espacios o servicios. Sin embargo, aquellas personas que no forman parte de esta mayoría encuentran dificultades en forma de barreras diversas, que impiden su participación como ciudadanos de pleno derecho (Ararteko 2011, Cayo y Lorenzo 2016, Observatorio Estatal de la Discapacidad 2016, Díaz Velázquez 2017). Todo lo cual nos aleja del objetivo de que los entornos deben ajustarse a la diversidad de las necesidades de la población y ser accesibles para todas las personas, permitiendo el ejercicio de todos y cada uno de sus derechos fundamentales.

En este contexto, a continuación este artículo se centrará en la situación de exclusión en la que se encuentran las personas sordas en el contexto español.

\section{Contextos de exclusión de las personas sordas en el Estado español}

La sordera afecta a más de un millón de personas en España, según datos de la última encuesta sobre discapacidades, autonomía personal y situaciones de dependencia del Instituto Nacional de Estadística $(\mathrm{INE})^{5}$. El $8 \%$ de la población posee problemas auditivos de distinto tipo y grado. Casi tres cuartas partes de este colectivo tiene más de 65 años. Y entre una y cinco de cada mil personas nacen con algún tipo de sordera.

Las personas sordas se encuentran en una situación de discapacidad socialmente desapercibida. Los entornos diseñados desde una cultura oral, en la que el acceso a la comunicación y a la información se transmite mediante las lenguas orales (en formato verbal o escrito), provocan situaciones de desventaja evidentes para el ejercicio de los derechos de estas personas. Las barreras de comunicación y acceso a la información que encuentran las personas sordas no

${ }^{5}$ La última encuesta sobre discapacidades, autonomía personal y situaciones de dependencia del INE fue llevada a cabo en al año 2008. Previamente se habían llevado a cabo otras dos, en 1986 y en 1999. Véase: https://www.ine.es/dyngs/INEbase/es/ categoria.htm?c=Estadistica_P\&cid=1254735573175 
son visibles a los ojos de la sociedad oyente que no vive ni conoce sus circunstancias (Díaz-Estebáñez 1996, Davis 2005, Muñoz-Baell 2011); de ahí la importancia que adquieren la toma de conciencia, la accesibilidad universal, las medidas contra la discriminación y las medidas de acción positiva, para lograr la eliminación de las barreras y garantizar el disfrute de sus derechos en las mismas condiciones que sus conciudadanos oyentes.

Las personas sordas no tienen características aparentemente visibles que evidencien sus particularidades y sus necesidades, y es habitual que otras personas no sean conscientes de las consecuencias de no tomar en cuenta sus especificidades en un entorno oyente, por lo que se generan barreras que provocan situaciones de exclusión, soledad e incomprensión. A continuación prestaremos atención a algunos de estos obstáculos a los que tienen que hacer frente.

\subsection{El desconocimiento de su realidad y de su identidad}

La primera barrera, por tanto, es el desconocimiento que hay entorno a las personas sordas (Sánchez Caballero 2017) y, para superarla, es necesario conocer su diversidad audiológica, lingüística e identitaria, y su realidad en los distintos ámbitos de la vida. En este sentido, y en función del grado de pérdida auditiva, es usual hacer una distinción entre sordera e hipoacusia: sordera, para referirse a personas con una sordera profunda que les impide aprender de forma natural el lenguaje oral y con una audición que no es funcional para la vida cotidiana ni para la adquisición de un lenguaje oral; e hipoacusia cuando, a pesar de tener una pérdida auditiva, tienen una audición más o menos funcional para la vida y, con o sin prótesis, adquieren el lenguaje por vía auditiva, aunque presenten mayores o menores incorrecciones de articulación, léxico y estructuración.

También hay que tener en cuenta que las necesidades de apoyo a la comunicación serán diferentes en función del momento de aparición de la sordera y del desarrollo lingüístico adquirido. En este sentido, la sordera es prelocutiva cuando se ha producido antes de haber adquirido el habla, independientemente de la calidad de su percepción; $y$, postlocutiva cuando ésta se produce después de haber adquirido el lenguaje ${ }^{6}$.

6 Para una información más detallada de la discapacidad auditiva y de la clasificación audiológica, véase la página web de la Confederación Española de Familias de Personas Sordas (www.fiapas.es), García Perales y Herrero (2008), o Salamanca y Guadalupe (2008). 
Con respecto al uso de los términos personas sordas o personas con discapacidad auditiva, hay que decir que tanto en la normativa como a nivel social se suelen utilizar indistintamente. Esto no obstante, la Confederación Estatal de Personas Sordas (CNSE), la Unión Europea de Personas Sordas (EUD) y Federación Mundial de Personas Sordas (WFD) suelen utilizar mayoritariamente el término personas sordas. En este artículo se utilizará asimismo el término persona sorda independientemente de si tiene una sordera profunda o una hipoacusia, o de si se trata de una sordera prelocutiva o postlocutiva.

Al igual que antes se ha expuesto en torno a la discapacidad, la conceptualización de la sordera también varía y se puede entender desde dos perspectivas: desde un enfoque médico, considerando la sordera física, que se centra en la etiología, el grado y tipo de pérdida, su localización, etc. y, desde un enfoque sociolingüístico-cultural, atendiendo a la lengua e identidad cultural de la persona. Este segundo enfoque hace hincapié en las consecuencias lingüísticas, culturales e identitarias de la sordera, y tiene en cuenta los diferentes procesos de socialización que se producen en ellas (de Vicente 2006, Salamanca y Guadalupe 2008).

En referencia a las características lingüísticas, puede afirmarse que las personas sordas son una minoría incomprendida con necesidades de comunicación únicas (McClelland, Chisholm y Powell 2001, McKee et al. 2012, CNSE 2013). Ahora bien, ni todas las personas sordas se comunican en lengua oral, ni todas ellas utilizan la lengua de signos. Hay personas sordas que tienen un buen dominio tanto de la lengua de signos como de la lengua oral, y hay personas que, teniendo un buen dominio de la lengua de signos, no tienen una buena competencia lingüística en la lengua oral (ni en su forma hablada ni escrita). Para las personas sordas que tienen un buen nivel de lenguaje oral, las prótesis y ayudas técnicas a la audición pueden ser suficientes para la participación en el mundo que les rodea; mientras que para otras, siendo necesarias, no son suficientes para el acceso a la información y la comunicación. También pueden manejarse mediante la lectoescritura, aunque esto no sea la solución en muchas ocasiones.

Las personas sordas que no dominan el lenguaje oral, por su parte, suelen presentar bajos niveles de comprensión lectora y expresión escrita, lo que les impide acceder a la información transmitida a través del leguaje escrito. Por eso, en muchas ocasiones no será efectivo el recurso de la lectoescritura para transmitir información a una persona sorda.

Por otro lado, existe un tópico muy generalizado en torno a la idea de que todas las personas sordas tienen unas habilidades asombrosas 
para la lectura labial y, por tanto, pueden comunicarse perfectamente. Sin embargo, ni todas las personas sordas tienen desarrollado el mismo nivel de destreza en la lectura labial ${ }^{7}$ ni todos los contextos son adecuados para ella ${ }^{8}$.

Otra actitud también generalizada cuando se habla a una persona sorda es la tendencia a gritar con la intención, consciente o no, de que va a poder oír o entender mejor el mensaje. No obstante, no sirve de nada subir la intensidad de la voz para que nos oiga (CNSE 2011 y 2014, Cañizares 2015).

Finalmente, cabe destacar que las personas sordas que se comunican en lengua oral constituyen en torno al $98 \%$ de la población sorda en España. Cierto es que el nivel de competencia lingüística es muy diferente entre quienes han perdido audición después de haber asentado el lenguaje y el habla y las personas que siempre han sido sordas. Entre esas últimas, pocas llegan a ser competentes en el idioma oral en un grado satisfactorio. Una prueba de ello, a falta de estudios rigurosos que nos permitan profundizar en el conocimiento de esta realidad, es el escaso número que, a día de hoy, ha alcanzado una titulación universitaria, habiendo necesitado incluso en algunos casos personal de apoyo al no tener plenas competencias de lecto-escritura.

Algunas personas con hipoacusia (unas 500.000, esto es, en torno al 14\%) utilizan prótesis auditivas (audífonos y/o implantes), gracias a las cuales tienen una audición funcional, y consideran la lengua oral como su lengua materna (Rouco, Vaamonde, del Río 2016). El porcentaje de personas con prótesis auditivas es mayor conforme aumenta el grado de pérdida de audición. Por otro lado, están las personas sordas signantes, que mayoritariamente no usan prótesis auditivas y que consideran la lengua de signos como su lengua materna, quienes conforman un colectivo de aproximadamente 13.300 personas en España (Martínez Tebar 2017). Un colectivo al que habría que añadir otras muchas personas signantes, hasta 100.000 en España según la OMS ${ }^{9}$, con diferentes niveles de dominio de la lengua de signos. Las personas sordas signantes, por tanto, conforman una minoría lingüística y tienen una experiencia común de vida que

7 Esta destreza requiere de mucha práctica, del dominio lingüístico y del conocimiento léxico del idioma utilizado.

8 Hay también una serie de factores no personales que pueden favorecer el óptimo desarrollo de la lectura labial, como son las condiciones lumínicas adecuadas, la ausencia de elementos distractores, etc.

9 Datos para el año 2005. Véase: www.visualfy.com/es/cuantas-personas-sordasusan-la-lengua-de-signos-en-europa/. 
se manifiesta en la cultura sorda (Glickman y Carey 1993). La cultura sorda incluye su historia y prácticas compartidas en lengua de signos, las creencias, normas, valores, actitudes, el humor, la poesía, las tradiciones literarias y el arte en todos sus formatos, que tratan sobre las experiencias de las personas sordas. Por ello debemos construir un entorno social que facilite la convivencia entre la cultura oyente y la cultura sorda. La identidad de la comunidad sorda se desarrolla en torno a su propia cultura expresada a través de la lengua de signos, y los miembros de las comunidades sordas en todo el mundo se identifican como miembros de un grupo cultural y lingüístico. Esta comunidad tiene estructurada una red de relaciones en torno a organizaciones y entidades donde la sordera no es considerada ni deficiencia ni discapacidad. Su seña de identidad es la lengua de signos, pero también su propia realidad socio-cultural, construida por las propias personas sordas signantes, su forma de vida, su comunicación holística y no lineal, su capacidad de síntesis, sus prácticas sociales y solidaridad, su visión del mundo y su mentalidad (CNSE 2013, WFD 2019a). El desarrollo de la identidad de las personas sordas no se define por el grado de pérdida auditiva, sino más bien por la identidad con el lenguaje, y ésta es una elección muy personal. A este respecto hay que tener en cuenta que ni todas las personas sordas se identifican con la cultura sorda, ni la comunidad sorda la conforman exclusivamente personas sordas.

Glickman y Carey (1993) fueron dos de los primeros investigadores en analizar el desarrollo de la identidad de las personas sordas. Para ello, elaboraron un instrumento para medir cómo se identifican las personas sordas con la comunidad sorda y su cultura, y determinaron cuatro tipos de identidades culturales con las que se identifican las personas sordas: a) identidad de la cultura oyente, b) identidad de la cultura sorda, c) identidad bicultural y, d) identidad marginal.

a) La identidad de la cultura oyente se refiere a actitudes y creencias de la cultura dominante, es decir, la cultura oyente, sobre la pérdida de audición. Estas personas se identifican con la comunidad oyente, se comunican en lengua oral y ven la sordera desde una perspectiva médica, como una discapacidad.

b) La identidad de la cultura sorda se refiere a actitudes y creencias de la cultura visual. Estas personas se identifican con la comunidad sorda, se comunican en lengua de signos, comparten su cultura y se relacionan con otras personas sordas.

c) Identidad bicultural: las personas sordas que han logrado cierto sentimiento de comodidad tanto en los entornos comunicativos 
auditivos (con personas oyentes) como visuales (con personas sordas).

d) Identidad marginal: esta orientación es típica de las personas que se sitúan entre el mundo sordo y el oyente, pero no se sienten cómodas en ninguno de los dos, o no están seguras de sus sentimientos hacia la sordera.

Como se puede observar, no todas las personas sordas son iguales, los factores personales (como la edad, el sexo o el nivel de formación), los factores audiológicos (como el grado y momento de la pérdida de audición), y los factores ambientales (como por ejemplo, las vivencias personales, actitudinales, relacionales, etc.), así como el contexto familiar, educativo y social, configuran la propia identidad personal (Bat-Chava 2000, Al-Makhamreh 2013, Chen 2014). Sin embargo, todas ellas comparten un denominador común: los aspectos visuales configuran, en mayor o menor medida, su contacto con el medio, y encuentran barreras de comunicación en su vida cotidiana. Para garantizar su participación en todas las áreas de la vida es necesario, por tanto, a) que se conozca, reconozca y apoye su identidad lingüística y cultural, sea ésta la lengua oral, la lengua de signos, la cultura auditiva o la cultura visual (Artículo 30.4 de la CDPD); b) que se conozca y respete en términos de dignidad la diversidad de las personas sordas; y, c) que se garantice el acceso a la información y comunicación en todos los ámbitos, reconociendo y respetando el uso del lenguaje.

\subsection{Dificultades de accesibilidad}

La segunda gran barrera que encuentran las personas sordas para el ejercicio de sus derechos fundamentales es la accesibilidad. Las barreras de comunicación conllevan la vulneración del ejercicio de los demás derechos, porque sin accesibilidad al derecho que se quiere ejercer no hay derecho, y se produce una situación de discriminación y exclusión (CERMI 2018). Para las personas sordas con la identidad de la cultura sorda, la lengua de signos es un requisito previo fundamental para la plena realización de sus derechos humanos (CERMI 2019, WFD 2018 y 2019b).

La CDPD, en su artículo 2, hace referencia a los distintos formatos que permiten el acceso a la comunicación a la diversidad de las personas sordas. Así, entre otros, se refiere al lenguaje escrito, los sistemas auditivos, el lenguaje sencillo, o la tecnología de la 
información y las comunicaciones de fácil acceso. Por otra parte, considera como «lenguaje» tanto el lenguaje oral como las lenguas de signos. Asimismo, en el artículo 30.4 contempla el reconocimiento y el apoyo a la identidad cultural y lingüística específica de las personas sordas, incluyendo la lengua de signos y la cultura sorda.

En el ámbito nacional español, el reconocimiento oficial de la lengua de signos se obtuvo en el año 2007 con la aprobación de la Ley 27/2007, de 23 de octubre, por la que se reconocen las lenguas de signos españolas y se regulan los medios de apoyo a la comunicación oral de las personas sordas, con discapacidad auditiva y sordociegas ${ }^{10}$. Esta ley da respuesta a las reivindicaciones del movimiento asociativo de las personas sordas y recoge su derecho a la comunicación. Por un lado, destaca el valor lingüístico y cultural de la lengua de signos y, por otro lado, regula los medios de apoyo a la comunicación oral de las personas sordas, con discapacidad auditiva y sordociegas. Los medios de apoyo a la comunicación oral son los recursos tecnológicos y las ayudas técnicas que facilitan a estas personas el acceso a la expresión verbal y escrita de la lengua oral, y favorecen una comunicación más plena con su entorno. De esta manera, la Ley 27/2007 pretende subsanar las barreras de acceso a la información y comunicación, respetando el principio de libertad de elección de la forma comunicativa de las personas sordas que conforman este colectivo tan diverso. En esta ley queda recogido que las personas sordas en muchos casos no pueden acceder a la información y a la comunicación con el entorno porque no disponen de los recursos de apoyo que necesitan para comunicarse mediante la lengua oral y/o, en el caso de las personas sordas signantes, porque no disponen de intérpretes de lengua de signos.

Doce años después, y a pesar de los avances, muchas personas sordas no disponen en la mayoría de las áreas de la vida de adaptaciones visuales y acústicas para recibir la información auditiva, ni de los medios de apoyo para la comunicación oral, ni de un servicio de interpretación a la lengua de signos, lo que conlleva que las personas sordas vivencien situaciones de discriminación en la formación, en el empleo, en los medios de comunicación, en el acceso a los servicios sociosanitarios o a la justicia, o en el disfrute de la cultura (Sánchez Caballero 2017, García 2017). Según el Observatorio Estatal de la Discapacidad (2016), siguen sin facilitarse los apoyos técnicos que contribuyan a la eliminación de las barreras de comunicación, tan

10 Ver BOE núm. 255, de 24 de octubre de 2007. 
necesaria para la inclusión social de las personas sordas, así como el reconocimiento y promoción de la utilización de la lengua de signos como una parte de la identidad lingüística y cultural de las personas sordas signantes, conforme a lo establecido en la CDPD.

A continuación, se detallan algunas situaciones en las que no se actúa en función de los principios generales, en las que el Estado no asegura el ejercicio de todos los derechos humanos y libertades fundamentales. En muchas ocasiones se producen situaciones de exclusión y discriminación, de falta de conciencia y se mantienen los estereotipos, prejuicios y las prácticas nocivas respecto de las personas sordas. En estas situaciones no se garantiza la accesibilidad y, se vulnera el derecho a la libertad de expresión y de opinión y de acceso a la comunicación, incumpliendo lo establecido en la CDPD, en la Ley 27/2007 o en el Real Decreto 1/2013.

Estas situaciones de discriminación vividas por personas sordas en su realidad cotidiana están relacionadas con el acceso al transporte, con la participación en la vida cultural, o en actividades recreativas y de esparcimiento, con la libertad de decisión para contraer matrimonio y fundar una familia, con la igualdad de oportunidades de formación a lo largo de la vida, con el acceso a la justicia, con el acceso al empleo o con el acceso a los servicios de emergencia y los servicios sanitarios.

En relación con el acceso al transporte, en el artículo 18 de la CPDP se establece el derecho a la libertad de desplazamiento de las personas con discapacidad. Sin embargo, en reiteradas ocasiones se ha denunciado la denegación de embarque a personas sordas alegando la necesidad, por motivos de seguridad, de viajar acompañadas de una persona oyente, ofreciendo como opciones la compra de un segundo billete para esa persona oyente acompañante, o la devolución del dinero (CERMI 2009 y 2018).

La Confederación Española de Familias de Personas Sordas (FIAPAS) también ha denunciado la falta de accesibilidad audiovisual en algunos trenes de larga distancia. En ambos casos se vulnera el derecho a la movilidad personal recogido en el artículo 20 de esta Convención, que insta a garantizar la movilidad personal en la forma y en el momento que la persona desee, disponiendo de dispositivos y tecnologías de apoyo a un costo asequible.

Por otra parte, aún no se han tomado las medidas establecidas en el artículo 30 de la CDPD para garantizar el acceso a la participación en la vida cultural en formatos accesibles, así como a lugares donde se ofrezcan representaciones o servicios culturales tales como teatros, museos, cines, etc., para facilitar su accesibilidad a las personas sordas, quienes no cuentan con dispositivos de apoyo a la audición, ni con 
subtitulación, audiodescripción ni lengua de signos (CERMI 2017 y 2019).

En referencia al derecho de todas las personas sordas en edad de contraer matrimonio, a casarse y fundar una familia sobre la base del consentimiento libre y pleno de los futuros cónyuges, recogido en el artículo 23 de la CDPD, cabe subrayar la limitación al mismo que supone la reforma del Código Civil introducida por la Ley 15/2015, de 2 de julio, de la Jurisdicción Voluntaria ${ }^{11}$, y que modifica el art. 56, que queda redactado así:

«Quienes deseen contraer matrimonio acreditarán previamente en acta o expediente tramitado conforme a la legislación del Registro Civil, que reúnen los requisitos de capacidad y la inexistencia de impedimentos o su dispensa, de acuerdo con lo previsto en este Código.

$\mathrm{Si}$ alguno de los contrayentes estuviere afectado por deficiencias mentales, intelectuales o sensoriales, se exigirá por el Secretario judicial, Notario, Encargado del Registro Civil o funcionario que tramite el acta o expediente, dictamen médico sobre su aptitud para prestar el consentimiento.»

Con esta reforma del Código Civil, no sólo se mantiene la discriminación que históricamente han padecido las personas con problemas de salud mental para contraer matrimonio, sino que esta vulneración de principios básicos de la Convención se extiende, además, a las personas con discapacidad sensorial.

Esta ley constituye una discriminación por motivo de discapacidad. Afortunadamente, en el año 2017, la Ley 4/2017, de 28 de junio, de modificación de la ley 15/2015, de 2 de julio, de la jurisdicción voluntaria ${ }^{12}$ va a modificar a su vez el art.56 y va a reconocer la capacidad jurídica de las personas con discapacidad según lo dispuesto en el artículo 23.1 de la CDPD: se reconoce el derecho de todas las personas con discapacidad en edad de contraer matrimonio, a casarse y fundar una familia sobre la base del consentimiento libre y pleno de los futuros cónyuges en igualdad de condiciones.

Respecto al derecho a una educación inclusiva y en igualdad de oportunidades a lo largo de toda la vida, sin discriminación y en igualdad de condiciones, en el art. 24 de la CDPD se establece que, en los casos en los que sea necesario, se hagan ajustes razonables

11 Ver BOE núm. 158, de 3 de julio de 2015.

12 Ver BOE núm. 154, de 29 de junio de 2017. 
en función de las necesidades individuales y se faciliten medidas de apoyo personalizadas. Sin embargo, en muchos casos no se facilita el aprendizaje de la lengua de signos ni se promociona la identidad lingüística de las personas sordas. Tampoco se asegura la educación en los lenguajes, los modos y medios de comunicación más apropiados para cada persona y en entornos que permitan alcanzar su máximo desarrollo académico y social.

Entre el alumnado con sordera, por una parte, son muchos los que utilizan prótesis auditivas y que requieren de recursos de apoyo a la audición y a la comunicación oral, como pueden ser, por ejemplo, las emisoras FM. En algunos casos la dotación de estos recursos, sin embargo, se demora sin una clara justificación. Y, en otros casos, el profesorado no considera necesario su uso o los utiliza solamente para impartir algunos contenidos. Por otra parte, también son muchas las personas que cursan sus estudios utilizando la lengua de signos como lengua vehicular en la enseñanza, y que también se encuentran con que, durante meses, no disponen de intérprete de lengua de signos, no quedando asegurado su acceso a la información y comunicación. Además, en la enseñanza secundaria o en la enseñanza postobligatoria no se dispone de intérprete, o no al menos durante toda la jornada lectiva. Tampoco se garantizan estas medidas en las escuelas de idiomas y en el aprendizaje de las lenguas orales extranjeras. Las escuelas de idiomas siguen sin ser accesibles para las personas sordas. En ellas, además de los obstáculos de acceso a la información y a la comunicación, los estudiantes sordos están obligados a superar también las pruebas concernientes a la escucha y el habla, lo que supone otra gran barrera a la hora de lograr la titulación (CERMI 2019).

En cuanto a la igualdad de oportunidades en el acceso al empleo, las personas sordas que utilizan prótesis auditivas tienen derecho a utilizarlas en las pruebas de acceso al empleo público (tanto orales como escritas) para que puedan realizarlas en igualdad de condiciones que las demás personas que se presentan. Sin embargo, el último informe de CERMI (2019) recoge denuncias de personas sordas usuarias de prótesis auditivas a las que se les ha impedido su uso a la hora de presentarse a unas oposiciones.

En relación al acceso a la justicia en igualdad de condiciones, tal y como se recoge en el artículo 122 de la CDPD, la FIAPAS ha recogido a lo largo de la última década quejas de personas sordas que comunican en lengua oral, que se han encontrado en la tesitura de un procedimiento judicial y no han podido disponer de los medios de apoyo a la comunicación oral necesarios en esa situación, con lo que ello supone de desprotección para esta parte de la ciudadanía (CERMI 
2012). Por otro lado, en el caso de las personas sordas signantes se incumplen leyes vigentes como la Ley 13/2009, de 3 de noviembre, de reforma de la legislación procesal para la implantación de la nueva Oficina judicia/13. Esta ley, en su art. 143, que hace referencia a la intervención de intérpretes, establece en el apartado 1 que: "cuando alguna persona que no conozca el castellano ni, en su caso, la lengua oficial propia de la Comunidad hubiese de ser interrogada o prestar alguna declaración, o cuando fuere preciso darle a conocer personalmente alguna resolución, el Secretario por medio de decreto podrá habilitar como intérprete a cualquier persona conocedora de la lengua de que se trate, exigiéndosele juramento o promesa de fiel traducción». Y en el apartado 2, haciendo referencia explícita a las personas sordas, establece que se nombrará al intérprete de lengua de signos adecuado en cada caso. También se incumple la Ley Orgánica 5/2015, de 27 de abril, por la que se modifican la Ley de Enjuiciamiento Criminal y la Ley Orgánica 6/1985, de 1 de julio, del Poder Judicial, para transponer la Directiva 2010/64/UE, de 20 de octubre de 2010, relativa al derecho a interpretación y a traducción en los procesos penales y la Directiva 2012/13/UE, de 22 de mayo de 2012, relativa al derecho a la información en los procesos penales ${ }^{14}$. Cierto es que se producen situaciones de claro incumplimiento de la accesibilidad por parte del sistema judicial, pero en numerosos casos son originadas por defectos de forma cometidos por los miembros de la abogacía. Y es que, aunque existe en teoría una garantía jurídica de intérprete de lengua de signos, muchos abogados y abogadas no siguen el procedimiento para solicitarlo de cara a la celebración de un juicio, por lo que el juzgado puede decidir la continuación del mismo sin intérprete. Y todo ello sin menoscabo de la necesaria mejora en la calidad de las interpretaciones.

Asimismo, existen casos en los que el incumplimiento de estas leyes contraviene el art. 17 de la CDPD y se generan situaciones de desprotección de la integridad física y mental de las personas sordas. Este incumplimiento queda recogido en la guía en relación a los derechos de las personas sordas y sordociegas en prisión elaborada recientemente por la Asociación Pro Derechos Humanos de Andalucía (APDHA 2019). El estudio llevado a cabo por esta asociación muestra que las personas sordas privadas de libertad están condenadas a la invisibilidad y la exclusión, debido a la falta de acceso a la comunicación en lengua de signos y a la falta de protocolos de

13 Ver BOE núm. 266, de 4 de noviembre de 2009.

14 Ver BOE núm. 101, de 28 de abril de 2015. 
apoyo a la comunicación oral. Así, presentan, por ejemplo, el caso de un preso sordo signante en un centro penitenciario andaluz, que durante diez años estuvo recibiendo órdenes o instrucciones orales que no podía comprender, sin opción de comunicarse en lengua de signos, quedando excluido del tratamiento penitenciario. Este preso no hacía actividades, no se comunicaba con su familia ni salía de permisos, no seguía el tratamiento médico, perdía citas médicas e, incluso, recibía sanciones por hechos que, por su sordera, no podía haber cometido. Esta persona sorda signante en el centro penitenciario no contaba con intérprete de lengua de signos (ILS) y nadie en la institución, ni de entre el funcionariado ni de entre los presos, conocía esta lengua (APDHA 2019 , 6). En vista de la situación de vulnerabilidad y aislamiento en la que se encuentran en la actualidad las personas sordas que están en prisión, Instituciones Penitenciarias, junto con entidades representativas de diversas discapacidades sensoriales, entre las que se encuentran la Federación de Asociaciones de Personas Sordociegas de España (FASOCIDE), FIAPAS, ONCE, Y CNSE, han firmado un protocolo de actuación con la finalidad de garantizar sus derechos (APDHA 2019).

Finalmente, la realidad de las personas sordas en el ámbito de la salud dista mucho de acercarse a las condiciones de acceso en igualdad de condiciones tal y como establece el artículo 25 de la CDPD. Históricamente la comunicación y las barreras lingüísticas han aislado a la comunidad sorda de la educación sanitaria, de los programas y los mensajes de salud, de las informaciones sanitarias trasmitidas por los medios de comunicación; todo lo cual afecta a su propia salud y a su acceso a los servicios sanitarios (McKee et al. 2011 y 2012, Steinberg et.al. 2006).

Por una parte, queda mucho por hacer para establecer protocolos y procedimientos accesibles para las personas sordas en situación de riesgo y emergencia. Los servicios de emergencias 112, a pesar de llevar ya 22 años de funcionamiento, todavía no son accesibles en todas las Comunidades Autónomas, veinticuatro horas al día, siete días a la semana, por lo que siguen sin brindar una asistencia a las personas sordas equivalente a la ofrecida a la población sin discapacidad. En algunas Comunidades Autónomas sí se ha puesto en marcha un sistema por el que las personas sordas pueden enviar mensajes de texto en caso de emergencia, pero para ello deben inscribirse previamente y permanecer en esa misma Comunidad Autónoma en la que se han registrado. Empero, el servicio no ha ofrecido la posibilidad de comunicarse en lengua de signos (CERMI 2019), aunque esta situación parece que va a cambiar próximamente, ya que el 24 de enero de 2020 entra en vigor el Real Decreto 734/2019, de 20 de diciembre, por el 
que se modifican directrices básicas de planificación de protección civil y planes estatales de protección civil para la mejora de la atención a las personas con discapacidad y a otros colectivos en situación de especial vulnerabilidad ante emergencias ${ }^{15}$. Una norma que obliga a garantizar la accesibilidad universal del teléfono de emergencias 112.

De otro lado, es necesario que la administración sanitaria facilite las informaciones y los materiales de preparación para emergencias en formatos accesibles para la lectura o en lengua de signos, ya que de otro modo no llegarán a la población sorda (Neuhauser et al. 2013). En la crisis del ébola, que marcó la actualidad sanitaria en España en el año 2014, por ejemplo, el gobierno no ofreció información accesible, siendo las propias entidades representativas de las personas sordas quienes tuvieron que llevar a cabo su adecuación para hacerla accesible para este colectivo. Y por ello se sigue reclamando la adopción de las medidas necesarias (utilización de subtítulos, lengua de signos, textos de lectura fácil, etc.) para que las campañas informativas y preventivas en materia de salud tengan en cuenta las necesidades comunicativas y de acceso a la información de las personas sordas, ya que, de lo contrario, no se garantiza que los servicios comunitarios para la población general estén también a disposición de las personas sordas (García 2017).

Queda un importante camino por recorrer en este sentido, aunque también se van produciendo avances, como la eliminación desde el año 2015 de las barreras de comunicación para las mujeres sordas en el teléfono 016 de atención a víctimas o posibles víctimas de violencia de género, a través primero del servicio de videointerpretación para personas sordas SVIsual, y actualmente a través de la plataforma Alba ${ }^{16}$. Unas iniciativas largamente demandadas y que suponen un importante avance en la igualdad de oportunidades de estas ciudadanas.

Por otra parte, en el ámbito sociosanitario, las personas sordas también tienen derecho a ser atendidas por los servicios sociales y de salud en igualdad de condiciones que el resto de la ciudadanía. Sin embargo, estos servicios no son siempre accesibles para ellas. Desde la aprobación de la Ley 27/2007, las personas sordas tienen el derecho a disponer de un servicio de interpretación en lengua de signos para comunicarse con el personal sanitario. Esto no obstante, en la mayoría de ocasiones, y especialmente en los servicios de urgencias, no hay esta posibilidad y la comunicación se lleva a cabo mediante la escritura y/o

15 Para más detalle véase: https://www.boe.es/eli/es/rd/2019/12/20/734

16 Para más información, véase: http://www.cnse.es/proyectoalba/quienes-somos. php 
la lectura labial. En estas circunstancias, se producen situaciones en las que ni hay consentimiento informado, ni una comunicación básica fiable entre médico y paciente, con el consiguiente riesgo para la salud de este último. Muchas personas sordas describen problemas de comunicación a la hora de pedir cita, describir sus síntomas, explicar los antecedentes médicos, comprender las explicaciones del diagnóstico, del tratamiento, etc. Estos problemas surgen debido a la ausencia de un servicio de intérprete para las visitas médicas en un plazo breve, por lo que la comunicación queda frecuentemente en manos de la mayor o menor voluntad del personal sociosanitario, quien muchas veces por desconocimiento se empeña en utilizar la comunicación escrita, o utiliza una terminología médica que los pacientes no llegan a comprender (Rodríguez Martín 2016, García 2017). Unas barreras lingüísticas y culturales para acceder y participar en los servicios existentes de promoción, tratamiento y recuperación de la salud que tienen serias consecuencias tanto emocionales como en la salud de las personas sordas (y especialmente de las embarazadas o en situación de parto y postparto), tal y como reconoce la propia OMS (2015).

Por otra parte, la comunicación y el aislamiento a los que frecuentemente se ven sometidas las personas sordas pueden derivar en problemas de salud mental. Unos problemas que también se ven favorecidos por las restricciones de acceso que experimentan a menudo a la atención médica general (Fellinger, Holzinger y Pollard 2012), así como por las barreras lingüísticas y culturales que enfrentan para acceder y participar en los propios programas de salud mental, debido a la falta de conocimiento dentro de este sector de las consideraciones específicas de las personas sordas, así como a la escasa aceptación por parte de estas últimas de sus problemas de salud mental (Toboso 2006).

Por todo ello, resulta imperioso avanzar en el camino hacia una mayor accesibilidad para las personas con discapacidad auditiva en el ámbito sociosanitario, implementando distintas recomendaciones ya largamente planteadas, como son: la ampliación de la prestación de servicios de interpretación, así como la mejora de la calidad de los mismos, la extensión de los distintos medios de apoyo a la comunicación oral que no pasen por la interpretación de lengua de signos (ej., la utilización de equipos de amplificación de sonido, textos en lectura fácil, etc.), el aumento de los servicios de asesoramiento a la población sorda, la asignación de más tiempo en las consultas, la extensión de la información preventiva, y la sensibilización a todo el personal sanitario sobre las características y necesidades de este colectivo. 
Los profesionales de la salud a menudo carecen de actitudes, conocimientos y aptitudes para abordar de forma adecuada las necesidades de las personas con discapacidad. Así queda recogido en el Informe Mundial sobre la Discapacidad (OMS 2011), que subraya al tiempo como prioritaria la necesidad de concienciación y formación sobre la discapacidad de los profesionales sanitarios. Una formación que debería contemplar el conocimiento de estrategias de comunicación basadas en la experiencia visual de las personas sordas, la localización y el acceso a los servicios de intérprete, o las características personales de la población sorda.

\section{A modo de conclusión}

La sordera, al igual que la discapacidad, ha pasado de ser considerada como un problema individual que afecta a algunas personas como consecuencia de una deficiencia, a ser considerada también desde una perspectiva de construcción social, resultante de la interacción entre las personas y el entorno físico, social, cultural e ideológico en el que conviven.

A lo largo de los últimos años, el enfoque biopsicosocial de la discapacidad en general, y de la sordera en particular, ha ido cobrando relevancia, así como su abordaje desde una perspectiva de reconocimiento de sus derechos fundamentales. Es decir, desde la consideración de la inapreciable dignidad de todos y cada uno de los seres humanos, el concepto de autonomía o de libre determinación que exige que cada persona sea el centro de todas las decisiones que le afecten, la igualdad inherente de todos independientemente de las diferencias y la ética de la solidaridad que la sociedad exige para sustentar la libertad de la persona con los apoyos sociales correspondientes, se va a plantear la necesidad de que las políticas públicas se centren en hacer desaparecer las barreras que impiden el acceso de las personas con discapacidades al sistema de libertades públicas que los derechos humanos contribuyen a preservar y promover. Se trata, en definitiva, de garantizar el reconocimiento de que las personas con discapacidad tienen un lugar en la sociedad por derecho propio, y que el logro de su independencia y de su participación en condiciones de auténtica igualdad no es sólo un objetivo deseable desde el punto de vista social sino, fundamentalmente, un derecho.

A lo largo de este artículo, así haya sido de forma breve, se han destacado importantes avances en la visibilidad y reconocimiento de 
las necesidades de la población sorda, tanto desde el punto de vista internacional como nacional, y desde el ámbito legal, de las políticas públicas, a través del reconocimiento de la necesidad de medidas que eliminen las barreras sociales o los prejuicios todavía existentes y dirigidos hacia este heterogéneo colectivo de personas. $Y$ todo ello con la finalidad de lograr su inclusión y participación social en todos los ámbitos de la vida.

Pero, aun así, también se han puesto de manifiesto importantes deficiencias todavía existentes en el conocimiento de su realidad y de su identidad, así como notables dificultades de accesibilidad en distintos ámbitos como el transporte, la participación en la vida cultural, en el acceso a una educación inclusiva y en igualdad de oportunidades, en el acceso a la justicia, en el ámbito sociosanitario...

Una realidad que nos lleva a subrayar que el proceso de garantizar que las personas sordas disfruten de sus derechos humanos avanza con lentitud. Se trata de una realidad que no podemos dejar de denunciar por las graves repercusiones que ello acarrea en el desarrollo de la vida de estas personas. La tarea de visibilizar a la población sorda y sus necesidades resulta, por tanto, imperiosa para ella, pero es también un objetivo imprescindible para el logro de una sociedad verdaderamente incluyente.

\section{Referencias bibliográficas}

Al-Makhamreh, Sahar. 2013. "Hearing the voices of young deaf people: Implications for social work practice in Jordan». International Social Work 59, n. 1: 47-59. https://doi.org/10.1177/0020872813499057.

Ararteko. 2011. Diagnóstico de accesibilidad del sistema de transporte público en la CAPV: Informe extraordinario de la institución del Ararteko al Parlamento Vasco. Vitoria-Gasteiz: Ararteko.

Asís, Rafael de. 2013. "Sobre el modelo social de la discapacidad: críticas y éxito». Working Papers El tiempo de los derechos, n. 1: 1-12.

Asís, Rafael de. 2014. Sobre el concepto de accesibilidad universal, Instituto de Derechos Humanos Bartolomé de las Casas. Universidad Carlos III, 17-24.

Asociación Pro Derechos Humanos de Andalucía (APDHA). 2019. Derechos de las Personas Sordas y Sordociegas en Prisión. Sevilla: APDHA. Acceso el 5 julio de 2019. https://www.apdha.org/devastadoras-condiciones-personassordas-sordociegas-prision/.

Barton, Len. 1998. Discapacidad y sociedad. Madrid: Morata.

Bat-Chava, Yael. 2000. "Diversity of deaf identities». American Annals of the Deaf 145, n. 5: 420-28. https://doi.org/10.1353/aad.2012.0176. 
Cañizares, Gema. 2015. Alumnos con déficit auditivo: Un nuevo método de enseñanza-aprendizaje. Madrid: Narcea.

Cayo, Luis y Rafael de Lorenzo (dir.). 2016. La Convención Internacional sobre los derechos de las personas con discapacidad - 2006/2016: Una década de vigencia. Madrid: Cinca. Acceso el 4 de junio de 2019. http:// www.fderechoydiscapacidad.es/wp-content/uploads/2016/12/Decada-devigencia.pdf

CERMI. 2009. Derechos humanos y discapacidad: Informe España 2008. Madrid: Cinca. Acceso el 4 de junio de 2019. https://www.cermi.es/sites/ default/files/docs/colecciones/DerechosHumanos2.pdf

CERMI. 2012. Derechos humanos y discapacidad. Informe España 2011. Madrid: Cinca. Acceso el 4 de junio de 2019. https://www.cermi.es/es/colecciones/ derechos-humanos-y-discapacidad-informe-espa\%C3\%B1a-2011

CERMI. 2017. Derechos Humanos y Discapacidad. Informe 2016. Madrid: Cinca. Acceso el 4 de junio de 2019. https://www.cermi.es/es/actualidad/ novedades/derechos-humanos-y-discapacidad-informe-espa\%C3\%B1a2016

CERMI. 2018. Derechos Humanos y Discapacidad. Informe España 2017. Madrid: Cinca. Acceso el 28 mayo 2019. https://www.cermi.es/es/colecciones/ derechos-humanos-y-discapacidad-informe-espa\%C3\%B1a-2017

CERMI. 2019. Derechos humanos y discapacidad. Informe España 2018. Madrid: Cinca. Acceso el 28 de mayo de 2019. https://www.cermi.es/es/colecciones/ derechos-humanos-y-discapacidad-informe-espa\%C3\%B1a-2018

Chen, Ge. 2014. «Influential factors of deaf identity development». Electronic Journal for Inclusive Education 3, n. 2: 1-12.

Confederación Estatal de Personas Sordas (CNSE). 2011. Dossier de Prensa. 75 años luchando por la igualdad de oportunidades para todas las personas. Por nuestros derechos básicos. Una organización de y para las Personas Sordas, Acceso el 4 de junio de 2019. https://www.cnse.es/

CNSE. 2013a. Personas sordas. Comunidad Sorda y Cultura Sorda. Acceso el 5 de julio de 2019. http://www.cnse.es/

CNSE. 2014. Plan de actuación de la Confederación Estatal de Personas Sordas (CNSE). Madrid: CNSE. http://www.empleo.gob.es/es/estrategia-empleojoven/logos/planes/Plan_Actuacixn_CNSE_523.pdf.

Davis, Ann N. 2005. «Invisible disability». Ethics 116, n. 1: 153-213.

Díaz-Estebáñez, Esther et al. 1996. Las personas sordas y su realidad social. Un estudio descriptivo. Madrid: Ministerio de Educación y Ciencia

Díaz Velázquez, Eduardo. 2017. El acceso a la condición de ciudadanía de las personas con discapacidad en España. Madrid: Cinca.

Fellinger, Johannes, Daniel Holzinger y Robert Pollard. 2012. «Mental health of deaf people». The Lancet 379, n. 9820: 1037-44. https://doi.org/10.1016/ S0140-6736(11)61143-4.

García Perales, Francisco J. y Joaquín Herrero (coords.). 2008. Manual de atención al alumnado con necesidades específicas de apoyo educativo derivadas de la discapacidad auditiva. Sevilla: Junta de Andalucía. Acceso el 5 de julio de 2019. http://redined.mecd.gob.es/xmlui/handle/11162/3176. 
García, Sonia. 2017. «La accesibilidad de las personas sordas al sistema sociosanitario». Balance sociosanitario de la dependencia y la discapacidad. Acceso el 5 de julio de 2019. https://www.balancesociosanitario.com/Laaccesibilidad-de-las-personas-sordas-al-sistema-sociosanitario_a5249.html.

Glickman, Neil S. y John C. Carey. 1993. "Measuring Deaf Cultural Identities: A Preliminary Investigation». Rehabilitation Psychology 38, n. 4: 275-83. https://doi.org/10.1037/h0080304.

Jaúdenes, Carmen (dir). 2009. Estudios sobre población con sordera en España. Madrid: FIAPAS

Jáudenes, Carmen y Begoña Gómez Nieto. 2010. «Información y comunicación accesible para personas con discapacidad auditiva en los espacios públicos urbanizados». En Accesibilidad en los espacios públicos urbanizados, coordinado por Fernando Alonso, 187-97. Madrid: Ministerio de Vivienda.

Joly, Eduardo D. 2002. "La discapacidad como construcción social». En Seminario Interdisciplinario Capacidades y diferencias. Buenos Aires: Universidad Nacional de La Plata.

Lidón, Leonor. 2016. La discapacidad en el espejo y en el cristal: derechos humanos, discapacidad y toma de conciencia, artículo 8 de la Convención Internacional sobre los derechos de las personas con discapacidad, un camino previo por recorrer. Madrid: Cinca.

Martínez Tebar, Laura. 2017. "La sordera no nos limita», EFE Salud, 29 de septiembre.

McClelland, Roy, Daniel Chisholm y Stephen Powell. 2001. «Mental health and deafness: An investigation of current residential services and service users throughout the UK». Journal of mental health 10, n. 6: 627-636. https:// doi.org/10.1080/09638230120041371.

McKee, Michael M., Steve L. Barnett, Robert C. Block y Thomas A. Pearson. 2011. "Impact of communication on preventive services among deaf American sign language users». American Journal of Preventive Medicine 41, n. 1: 75-79. https://doi.org/10.1016/j.amepre.2011.03.004.

McKee, Michael, Denise Thew, Matthew Starr, Poorna Kushalnagar, John T. Reid, Patrick Graybill, Julia Velasquez y Thomas Pearson. 2012. «Engaging the deaf American sign language community: Lessons from a community-based participatory research center». Progress in Community Health Partnerships 6, n. 3: 321-29.

Muñoz-Baell, Irma M. et al. 2011. "Comunidades sordas: ¿pacientes o ciudadanas?» Gaceta Sanitaria, 25, n. 1: 72-78

Neuhauser, Linda, Susan Ivey, Debbie Huang, Alina Engelman, Winsto Tseng, Donna Dahrouge, Sidhanta Gurung y Melissa Kealey. 2013. "Availability and readability of emergency preparedness materials for deaf and hard-ofhearing and older adult populations: issues and assessments». PLOS ONE 8, n. 2: 1-11. https://doi.org/10.1371/journal.pone.0055614.

Observatorio Estatal de la Discapacidad. 2016. 2006-2016: 10 años de la Convención Internacional sobre los Derechos de las Personas con Discapacidad - Balance de su aplicación en España. Madrid: Observatorio 
Estatal de la Discapacidad. Acceso el 4 de junio de 2019. https:// observatoriodeladiscapacidad.info/attachments/article/85/BALANCE \%20 10\%20CONVENCION.pdf.

Organización Mundial de la Salud (OMS). 2001. Clasificación Internacional del Funcionamiento, de la Discapacidad y de la Salud. Ginebra: OMS.

OMS. 2011. Informe mundial sobre la discapacidad 2011. Ginebra: OMS.

OMS. 2015. "Sordera y pérdida de la audición». Nota descriptiva n. 300. Acceso el 5 de julio de 2019. http://www.who.int/mediacentre/factsheets/fs300/es/.

Padilla, Andrea. 2010. "Discapacidad: contexto, concepto y modelos». International Law, 16: 381-414.

Palacios, Agustina. 2008. El modelo social de discapacidad: orígenes, caracterización y plasmación en la Convención Internacional sobre los Derechos de las Personas con Discapacidad. Madrid: Cinca.

Palacios, Agustina y Javier Romañach. 2006. El modelo de la diversidad: La bioética y los derechos humanos como herramientas para alcanzar la plena dignidad en la diversidad funcional. A Coruña: Diversitas-AIES.

Palacios, Agustina y Javier Romañach. 2008. «El modelo de la diversidad: una nueva visión de la bioética desde la perspectiva de las personas con diversidad funcional (discapacidad)». Intersticios. Revista sociológica de pensamiento crítico 2, n. 2: 37-47.

Rodríguez Martín, Dolors. 2016. ¿Discapacitado? No, iSordo! Tesis doctoral defendida en la Universidad Autònoma de Barcelona. Acceso el 23 de junio de 2019. http://hdl.handle.net/ 10803/399217

Romañach, Javier. 2009. Bioética al otro lado del espejo: la visión de las personas con diversidad funcional y el respeto a los derechos humanos. A Coruña: Diversitas-AIES.

Rouco, J.M., I. Vaamonde y M. del Río. 2016. Audioprótesis external. Indicaciones. Selección del tipo de adaptación. Adaptación y evaluación de resultados. Rehabilitación del paciente adaptado con prótesis, en Libro virtual de formación en ORL. A Coruña: Complejo Hospitalario Universitario de Santiago.

Salamanca, Marcelo y Carolina Guadalupe. 2008. «Psicoterapia en familias con miembros sordos: Un modelo sistémico». Cultura Sorda. Acceso el 5 de julio de 2019. http://www.cultura-sorda.org/wp-content/uploads/2015/03/ Salamanca_Picon_Psicoterapia_sistemica_sordos_2008.pdf.

Sánchez Caballero, Daniel. 2017. "La falta de recursos lastra la ley que reconoció la lengua de signos tras diez años desde su aprobación». eldiario.es. 27 de octubre. Acceso el 5 de julio de 2019. https://www. eldiario.es/sociedad/asociaciones-personas-denuncian-incumpleinstauro_0_701330914.html

Steinberg, Annie G., Steven Barnett, Helen E. Meador, Erin A. Wiggins y Philip Zazove. 2006. "Health care system accessibility». Journal of General Internal Medicine 21, n. 3: 260-66. https://doi.org/10.1111/ j.1525-1497.2006.00340.x.

Susinos, Teresa. 2006. «Mujeres (dis)capacitadas o la construcción de un yo deficitario e incompleto». En Mujeres en la periferia: Algunos debates 
sobre género y exclusión social, editado por Adelina Calvo, Marta García y Teresa Susinos, 95-120. Barcelona: Icaria.

Toboso, Mario y Francisco Guzmán. 2010. "Cuerpos, capacidades, exigencias funcionales... y otros lechos de Procusto». Política y Sociedad 47, n. 1: 67-83.

Toboso, Remedios. 2006. «Unidad de Salud Mental para Personas Sordas (USMS) ». Trabajo Social Hoy 47: 7-21.

Vicente, Ana de. 2006. «Sordera y exclusión», en Exclusión social y estado de bienestar en España, editado por Fernando Vidal, 479-489. Madrid: Icaria.

World Federation of the Deaf (WFD). 2018. "International Day of Sign Languages and International Week of the Deaf 2018». World Federation of the Deaf (blog). Acceso el 5 de julio de 2019. http://wfdeaf.org/news/ theme-for-international-day-of-sign-languages-and-international-week-ofthe-deaf-2018/.

WFD. 2019a. "7 August 2019: WFD Position Paper on the Complementary or diametrically opposed: Situating deaf communities within 'disability' vs cultural and linguistic minority' constructs». World Federation of the Deaf (blog). Acceso el 5 de julio de 2019. http://wfdeaf.org/news/resources/ wfd-position-paper-complementary-diametrically-opposed-situating-deafcommunities-within-disability-vs-cultural-linguistic-minority-constructs/.

WFD. 2019b. «International week of the Deaf 2019». WFD. World Federation of the Deaf (blog). Acceso el 5 de julio de 2019. https://wfdeaf.org/iwdeaf2019/.

Zola, Irving K. 2005. "Toward the necessary universalizing of a disability policy». The Milbank Quarterly. A Multidisciplinary Journal of Population Health and Health Policy 83, n. 4. https://doi.org/10.1111/j.1468-0009.2005.00436.x. 


\section{Copyright}

Deusto Journal of Human Rights / Revista Deusto de Derechos Humanos is an Open Access journal; which means that it is free for full and immediate access, reading, search, download, distribution, and reuse in any medium only for non-commercial purposes and in accordance with any applicable copyright legislation, without prior permission from the copyright holder (University of Deusto) or the author; provided the original work and publication source are properly cited (Issue number, year, pages and DOI if applicable) and any changes to the original are clearly indicated. Any other use of its content in any medium or format, now known or developed in the future, requires prior written permission of the copyright holder.

\section{Derechos de autoría}

Deusto Journal of Human Rights / Revista Deusto de Derechos Humanos es una revista de Acceso Abierto; lo que significa que es de libre acceso en su integridad inmediatamente después de la publicación de cada número. Se permite su lectura, la búsqueda, descarga, distribución y reutilización en cualquier tipo de soporte sólo para fines no comerciales y según lo previsto por la ley; sin la previa autorización de la Editorial (Universidad de Deusto) o la persona autora, siempre que la obra original sea debidamente citada (número, año, páginas y DOI si procede) y cualquier cambio en el original esté claramente indicado. Cualquier otro uso de su contenido en cualquier medio o formato, ahora conocido o desarrollado en el futuro, requiere el permiso previo por escrito de la persona titular de los derechos de autoría. 